\title{
Digital Asymmetric Waveform Isolation (DAWI) in a Digital Linear Ion Trap
}

\author{
Francesco L. Brancia, ${ }^{a}$ Bryan McCullough, ${ }^{\text {b }}$ Andrew Entwistle, ${ }^{c}$ \\ J. Günter Grossmann, ${ }^{\mathrm{d}}$ and Li Ding ${ }^{\mathrm{e}}$ \\ ${ }^{a}$ Faculty of Agricultural Science, Aarhus University, Aarhus, Denmark \\ ${ }^{\mathrm{b}}$ Michael Barber Centre for Mass Spectrometry, University of Manchester, Manchester, United Kingdom \\ ' Shimadzu Research Laboratory (Europe), Manchester, United Kingdom \\ d School of Biological Sciences, University of Liverpool, Liverpool, United Kingdom \\ e Shimadzu Research Laboratory, Shanghai, China
}

Traditionally, in a quadrupole mass filter, ion isolation is achieved by scanning the rf and DC voltages with a fixed ratio. In this paper, we describe an innovative procedure implemented in a digitally driven linear ion trap termed digital asymmetric waveform isolation (DAWI) in which ion isolation is obtained by manipulation of the duty cycle of the rectangular waveforms. Variation of the waveform duty cycle allows introduction of a precisely defined DC quadrupole component into the main trapping field of the quadrupole ion filter. The DAWI method is completely controlled at software level and does not require any hardware modification. (J Am Soc Mass Spectrom 2010, 21, 1530-1533) (c) 2010 Published by Elsevier Inc. on behalf of American Society for Mass Spectrometry

$\mathrm{U}$ nlike amplitude driven quadrupole ion traps, the three-dimensional (3D) digital ion trap (DIT) mass spectrometer has been designed bearing in mind that instead of using sinusoidal waveforms, rectangular waveforms could be employed to drive the ion trap [1, 2]. In the DIT, the quadrupole trapping and excitation waveforms are generated by rapidly switching between discrete DC voltage levels using fast high voltage electronics. As consequence of this, mass spectra can be obtained by means of frequency scan instead of conventional voltage scan, thus extending the mass range up to the MALDI analysis of intact proteins [3-6]. Unique features related to the type of waveforms used include high precursor ion isolation using a combination of forward and reverse scans [4] and the ability to perform electron capture dissociation (ECD) without the necessity of utilizing a permanent magnetic field [5]. Amongst all these unique advantages, the rectangular waveforms can be manipulated at software level so that a well-defined DC component can be superimposed on the main trapping field by variation of the waveform duty cycle. This approach has been recently used to determine experimentally the $\beta_{\mathrm{r}}$ and $\beta_{z}$ boundary lines of the 3D digital ion trap stability diagram using several peptides. Using this approach, several duty cycle values corresponding to different working points theoretically predicted for crossing the boundary of the stability region are chosen and the experimental stability diagram is produced [7].

Address reprint requests to Dr. F. L. Brancia, Department of Food Science, Faculty of Agricultural Science, Aarhus University, Blichers Alle, P.O. Box 50, 8830 Tjele, Denmark. E-mail: Francesco.Brancia@agrsci.dk
Since its invention [8], the quadrupole mass filter has become a constitutive element present in a vast range of commercially available mass spectrometers. To isolate ions within a well-defined $\mathrm{m} / \mathrm{z}$ range, a new mass scan line differing from $\mathrm{q}=0$ has to be generated superimposing a DC component on the main trapping field. To do so, an additional DC power supplier needs to be added. In the early 1970s, Richards and coworkers suggested the utilization of rectangular waveforms to perform ion isolation in a quadrupole mass filter. However, due to the impossibility to ionize large biomolecules at the time and the limitations of the electronics used, the paper provided only the proof-of-principle rather than a real analytical method applicable to any type of analytical samples [9]. In this paper, we show that in a linear digital ion trap (DLIT), using two pairs of switches that generate two opposite phases of digital waveform for the $x$-poles and $y$-poles, respectively, variation of the waveform duty cycle controlled at software level provides a fast method, termed digital asymmetric waveform isolation (DAWI), which can be employed for ion isolation.

\section{Methods}

\section{Mass Spectrometry}

The instrument consists of a 3D digital ion trap (DIT) mass spectrometer based on the design published previously [10]. It comprises an off-axis microspray source. Cytochrome $c$ (Sigma, Poole, UK) was dissolved in 50\% ( $\mathrm{vol} / \mathrm{vol})$ acetonitrile acidified with $0.1 \%$ (vol/vol) formic acid and sprayed at flow rates of $10 \mu \mathrm{L} / \mathrm{min}$ with a 


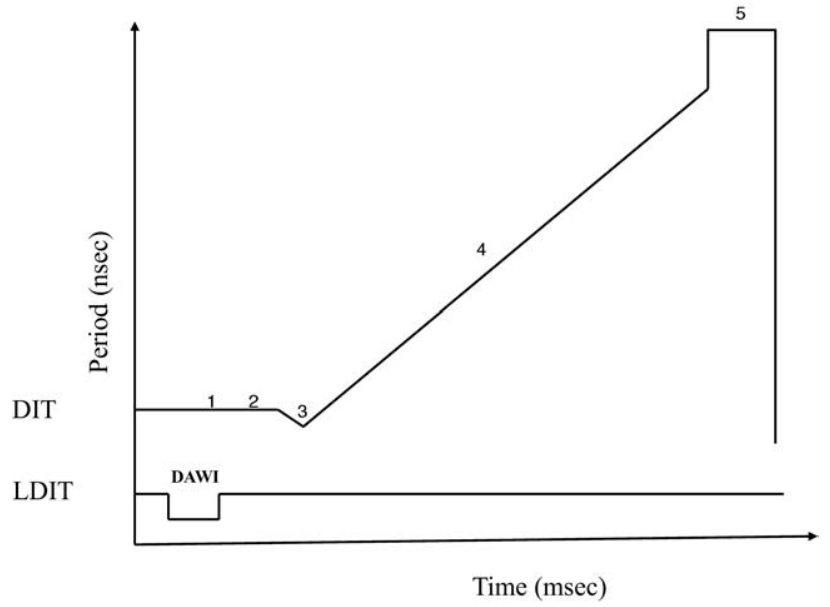

Figure 1. The scan tables used for both DIT and LDIT to perform DAWI. When DAWI is performed in the section B of the LDIT, the potential is lowered in section $C$, and ions are transmitted and trapped in the DIT for mass analysis. Following the DAWI step, a $5 \mathrm{~V}$ bias is applied to $C$ again and ions are not allowed in this section till the next experiment. In the DIT scan table, ion trapping following DAWI (1), ion cooling (2), field adjusting (3), mass scan (4), and standby mode (5) are indicated. The scale for each operation step is arbitrary.

concentration of $5 \mathrm{pmol} / \mu \mathrm{L}$. A 'Q-array' (an rf ion guide consisting of three sets of electrode plates) transports the ions (via a skimmer) to the digital linear quadrupole ion trap before injection into the 3D trap. The linear ion trap is segmented in three distinct sections of $20 \mathrm{~mm}(\mathrm{~A}), 84 \mathrm{~mm}(\mathrm{~B})$, and $6 \mathrm{~mm}(\mathrm{C})$, respectively. Diameter of the rods is only $6 \mathrm{~mm}$ for all three sections. Both rf ion guides $(\mathrm{Q}$ array and linear quadrupole) are digitally driven using parameters that can be defined separately in the control software. Voltages applied to each of the linear ion trap segments can be varied to perform different functions within the device (ion storage and DAWI). Figure 1 displays the scan table with the voltages applied to both DLIT and 3D DIT. Ions are then pulsed into the 3D trap by lowering the potential on the gate electrode for a user-defined time (typically a few $\mu$ s). In the 3D DIT a mass spectrum is generated by resonance ejection. Ion detection is then performed on a channel electron multiplier (Burle/Photonis, Lancaster, PA, USA) with a conversion dynode. Spectra are processed using our in-house built software.

\section{Results}

In the DIT mass spectrometer, the 3D digital ion trap is used as mass analyzer to perform all main analytical functions (mass analysis, precursor ion isolation, ion activation) while the digitally driven linear ion trap

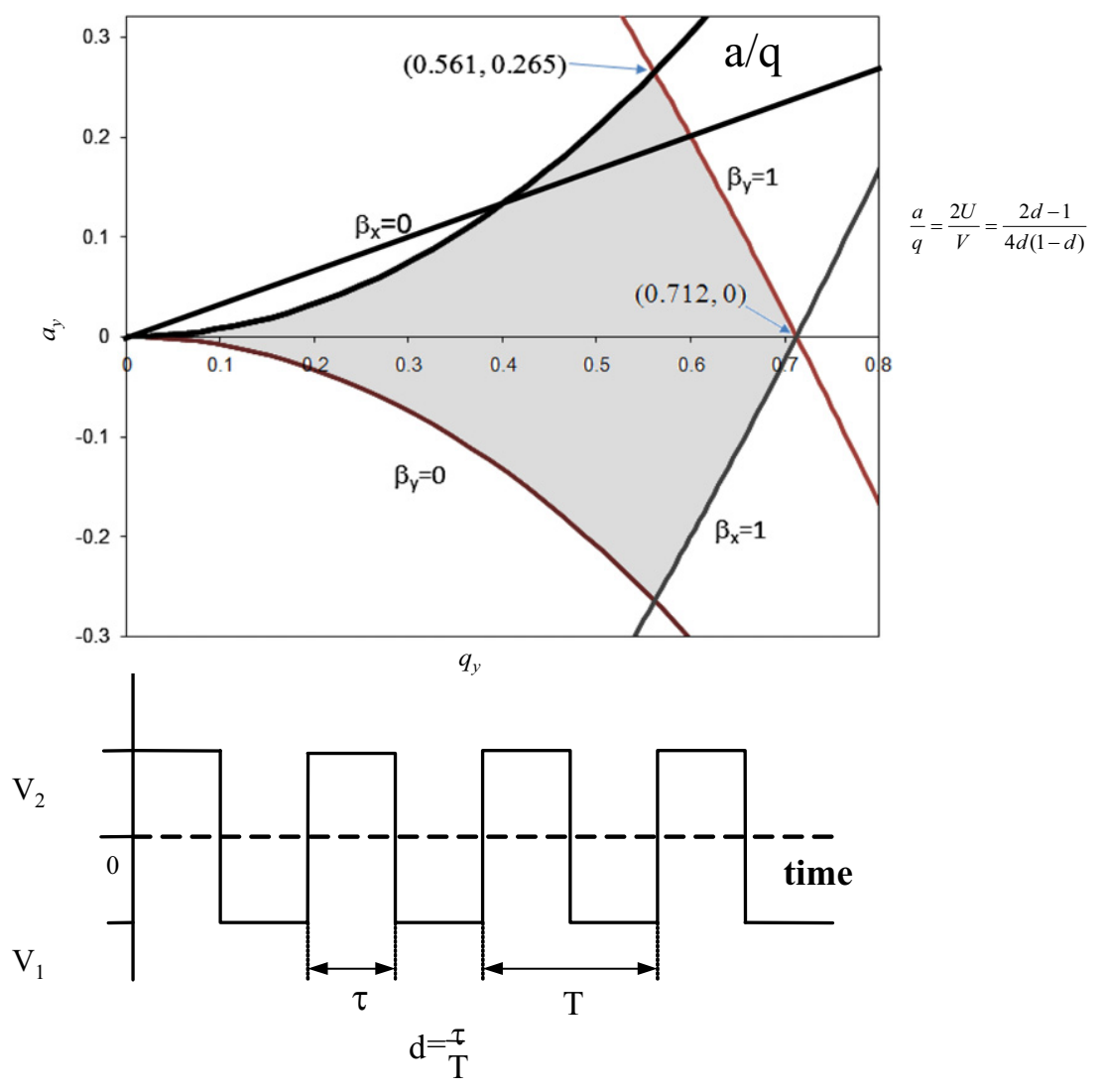

Figure 2. The stability diagram for the digital linear ion trap is plotted for the case of equal plus and minus voltage levels, in which the resolving DC level is created only using an asymmetric duty cycle waveform between the $x$ - and $y$-poles. The slope of the straight line, which is the ratio between $\mathrm{U}$ and $\mathrm{V}$, can be described as function of $\mathrm{d}$. In the lower figure, $d$ is defined as function of the period T. 
positioned before the DIT is employed exclusively for ion focusing and ion selection. Here, we want to show how the use of digital drive for the linear ion trap can facilitate its utilization as mass filter removing the necessity of an additional DC power supplier. Similar to the DIT, the linear ion trap described here is driven using rectangular waveforms. Using two pairs of switches that generate two opposite phases of digital waveform for the $x$-poles and $y$-poles, respectively, the radial confinement electric field is generated. Confinement along the axis of the linear ion trap can be achieved using two end-rods (sections A and C) so that an axial potential barrier is produced. The stability diagram for the digital linear ion trap, displayed in Figure 2, can be obtained with the same theoretical method used for the 3D ion trap [11]. The diagram is plotted for the case of equal, plus, and minus voltage levels, in which case the resolving DC level is created only using an asymmetric duty cycle waveform between the $x$ - and $y$-poles. In absence of any additional DC quadrupole component, the working points of all ions sit on the $q_{z}$-axis, and $q_{z}=0.712$ is the low mass cut-off point corresponding to the stability boundaries in both the $x$ - and $y$-directions. The apex of the first stability region is located at $q_{y}=0.561, a_{y}=0.265$. In the steady trapping operation, a periodic rectangular wave voltage is generated by switching between a high voltage level $V_{1}$ and a low voltage level $V_{2}$. The $U$ and $\mathrm{V}$ values are function of the average values of the DC and AC components and the duty cycle $d$ (ratio between $\tau$ and the total period $\mathrm{T}$ of the wave employed) of the rectangular wave voltage applied to the $x$ and $y$ rods as indicated in the two equations below:

$$
\begin{aligned}
& U=d \mathrm{~V}_{1}+(1-d) \mathrm{V}_{2} \\
& \mathrm{~V}=2\left(\mathrm{~V}_{1}-\mathrm{V}_{2}\right)(1-d) d
\end{aligned}
$$

To operate the linear ion trap as a mass filter ions are isolated by scanning the $\mathrm{rf}$ and DC voltages with a fixed ratio. In the $a, q$ cartesian space, the slope of the straight line $2 U / V$ can be expressed as function of the duty cycle $d$ :

$$
\frac{a}{q}=\frac{2 U}{V}=\frac{2 d-1}{4 d(1-d)}
$$

It is important to point out that the linear ion trap is segmented in three sections of different lengths. In the DAWI procedure described here, only section $B$ of the linear ion trap operates as mass filter. Following DAWI in section $B$, ions are stored in section $C$ before being injected in the 3D DIT for mass analysis. The DC axial potential bias applied on sections $\mathrm{A}, \mathrm{B}$, and $\mathrm{C}$ are provided separately. The smallest isolation window achievable (highest precursor ion resolution) is obtained when the $a / q$ line is very close to the apex of the first stability $\left(q_{y}=0.561, a_{y}=0.265\right)$. Under these conditions, the maximum value of duty cycle that can be used in the experiments is 0.612 . Figure 3 shows the
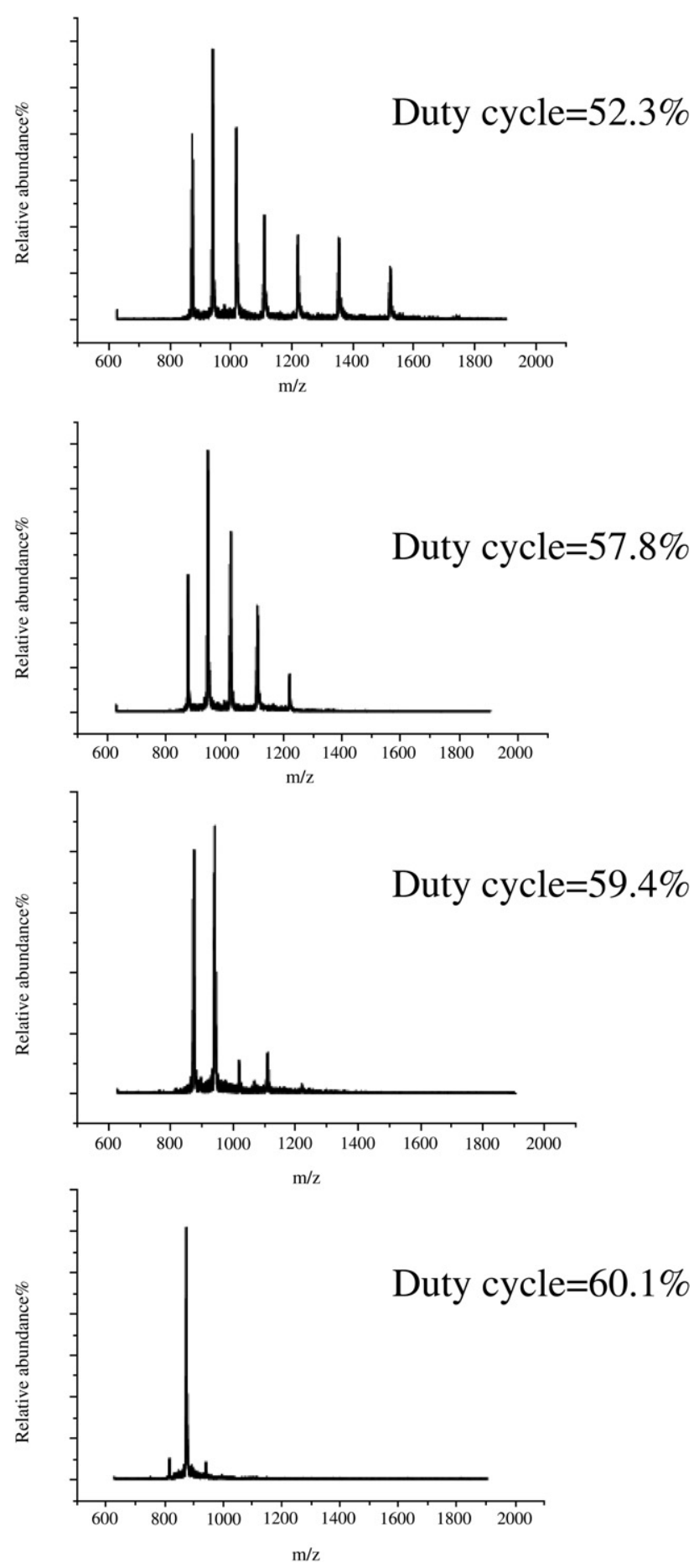

Figure 3. Electrospray mass spectra of cytochrome $c$ acquired varying the duty cycle from $50 \%$ up to $61.2 \%$. When the a/q approaches the apex of the stability diagram only the protein ions of charge state +13 at $\mathrm{m} / \mathrm{z} 942$ are isolated in the linear ion trap and subsequently transmitted to the 3D DIT for mass analysis by resonant ejection.

ESI mass spectra of cytochrome $c$ acquired using different duty cycle values applied to section B of the linear ion trap. In all experiments the quadrupole is set to transmit the $[\mathrm{M}+13 \mathrm{H}]^{13+}$ cytochrome $c$ ions at $m / z 942$. When no DC resolving component is used $(d=50 \%)$, 


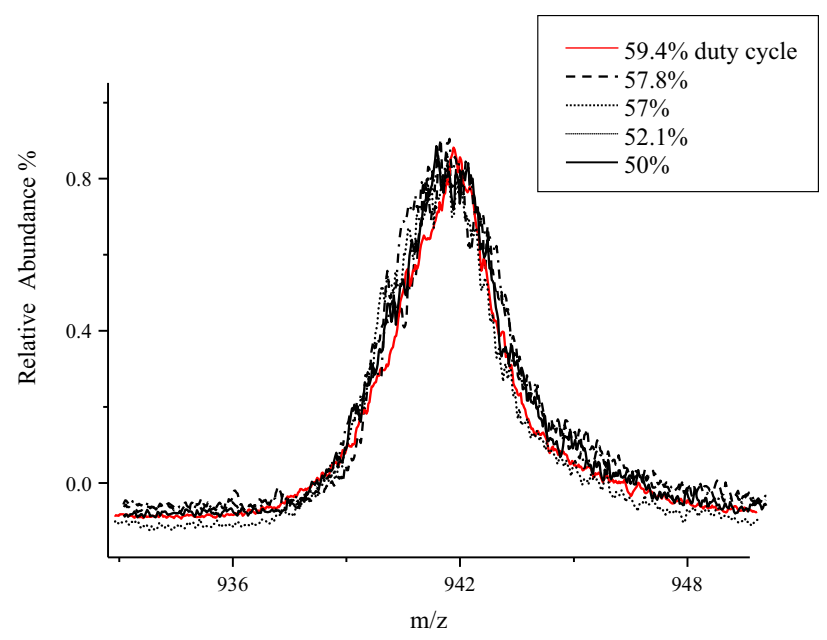

Figure 4. Ion abundances of cytochrome $[\mathrm{M}+13 \mathrm{H}]^{13+}$ acquired using different duty cycles.

the mass spectrum shows a mass range between 600 and 1900 amu. DIT mass analysis by resonant ejection covers the same mass range. Increasing the duty cycle up to $59.4 \%$ generates a spectrum in which it is still possible to detect protein ions of different charge state in proximity to the ion of interest. Isolation of the [M + $13 \mathrm{H}]^{13+}$ protein ions with concomitant removal of all other charge states is obtained only when $d$ is $60.1 \%$ (resolution around 100 FWHM). Due to software limitation, it is important to bear in mind that the duty cycle can be only varied by an increase of $0.7 \%$ duty cycle. During the DAWI isolation step in section B, section C is used to store the selected ion population for a defined time ( $8 \mathrm{~ms})$. To do so, ion confinement in section $C$ is obtained applying a DC bias of $5 \mathrm{~V}$ that is lowered for ion transmission into the DIT. After that the bias is applied again and ions are not allowed in this section till the next scan (see Figure 1). Using this experimental set-up, we also tried to determine the effect of higher duty cycle (higher precursor ion resolution) on isolation efficiency. Figure 4 shows the relative abundances of the multiply charged cytochrome $c$ ions under study acquired using different duty cycles. From the spectra obtained, no variation in isolation efficiency is reported when a higher precursor ion isolation resolution is observed.

\section{Conclusions}

Previous experiments carried out in our laboratory demonstrated how the utilization of a resolving DC component generated by varying the duty cycle of the rectangular waveform can be used as an alternative method for fast precursor ion isolation in a $3 \mathrm{D}$ digital ion trap $[12,13]$. Here we extend our approach to a linear ion trap showing that it can be used as a mass filter by simply varying the duty cycle of the rectangular waveform applied to the four rods. With respect to early works for analysis of krypton published nearly 40 years ago [9], we showed that efficient ion isolation of a specific electrosprayed charge state of a protein can be carried out before ion trapping in a 3D digital ion trap. The method can be controlled by only modifying the parameters entered in the control software and does not require an additional DC power supplier. The procedure has recently shown its utility in the attempt to perform gas-phase small angle X-ray scattering in a DIT mass spectrometer interfaced with a synchrotron radiation source [10]. However, due to its simplicity, DAWI could be implemented in any mass spectrometer.

\section{Acknowledgments}

The authors acknowledge Ikuo Konishi for the helpful discussions. The authors gratefully acknowledge the financial support from Shimadzu Corporation in funding this work. This work is also funded by the European Union FP6 Design Study "SmallAngle X-ray Scattering Initiative for Europe" (SAXIER), RIDS 011934.

\section{References}

1. Ding, L.; Sudakov, M.; Kumashiro, S. A Simulation Study of the Digital Ion Trap Mass Spectrometer. Int. J. Mass Spectrom. 2002, 221, 117-138.

2. Ding, L.; Kumashiro, S. Ion Motion in the Rectangular Wave Quadrupole Field and Digital Operation Mode of a Quadrupole Ion Trap Mass Spectrometer. Rapid Commun. Mass Spectrom. 2006, 20(1), 3-8.

3. Ding, L.; Sudakov, M.; Brancia, F. L.; Giles, R.; Kumashiro, S. A Digital Ion Trap Mass Spectrometer Coupled with Atmospheric Pressure Ion Sources. J. Mass Spectrom. 2004, 39(5), 471-484.

4. Brancia, F. L.; Giles, R.; Ding, L. Effect of Reverse Scan on Mass Measurement Accuracy in an Ion Trap Mass Spectrometer. J. Mass Spectrom. 2004, 39(6), 702-704.

5. Ding, L.; Brancia, F. L. Electron Capture Dissociation in a Digital Ion Trap Mass Spectrometer. Anal. Chem. 2006, 78(6), 1995-2000.

6. Tanaka, K.; Sekiya, S.; Jinno, M.; Hazama, M.; Kodera, K.; Iwamoto S. Analysis of Macromolecular Ions Using a MALDI DIT MS Mass Spectrometer. Proceedings 56th ASMS Conference; 2008 Denver, CO, June, 2008

7. Berton, A.; Traldi, P.; Ding, L.; Brancia, F. L. Mapping the Stability Diagram of a Digital Ion Trap (DIT) Mass Spectrometer Varying the Duty Cycle of the Trapping Rectangular Waveform. J. Am. Soc. Mass Spectrom. 2008, 19(4), 620-625.

8. Paul, W.; Reinhard, H. P. ; von Zahn, U. Das elektrische Massenfilter als Massenspektrometer und Isotopentrenner Zeitschrift fur Physik. 1958, 152, 143-182.

9. Richards, J. A.; Huey, R. M.; Hiller, J. A New Operating Mode for the Quadrupole Mass Filter. Int. J. Mass Spectrom. Ion Phys. 1973, 12, 317-339.

10. McCullough, B. J.; Entwistle, A.; Konishi, I.; Buffey, S.; Hasnain, S. S.; Brancia, F. L.; Grossmann, J. G.; Gaskell, S. J. Digital Ion Trap Mass Spectrometer for Probing the Structure of Biological Macromolecules by Gas Phase X-Ray Scattering. Anal. Chem. 2009, 81(9), 3392-3397.

11. Brancia, F. L.; Ding, L. Rectangular Waveform Driven Digital Ion Trap (DIT) Mass Spectrometer. In Practical Aspects of Trapped Ion Mass Spectrometry, Vol. IV: Theory and Instrumentation. March R. E., Todd J. F. J., Eds.; Taylor and Francis: London, 2010; pp 101-130.

12. Brancia, F. L.; Raveane, L.; Berton, A.; Traldi, P. Boundary-Activated Dissociation in a Digital Ion Trap. In Practical Aspects of Ion Trap Mass Spectrometry; Todd, J. F. J., March, R. E., Eds.; Taylor and Francis: London, 2009; pp 3069-3076.

13. Ding, L.; Brancia, F. L.; Giles, R.; Smirnov, S.; Nikolaev, E. Advances of Tandem Mass Spectrometrical Functions in Digital Ion Trap: High Resolution Ion Isolation and Electron Capture Dissociation. Proceedings 53rd ASMS Conference; San Antonio, TX, June 2005 